\title{
Mitteilung des Präsidenten der DGKFO
}

\section{Jörg Lisson ${ }^{1}$}

Angenommen: 15. Juni 2021

(c) Springer Medizin Verlag GmbH, ein Teil von Springer Nature 2021

Sehr geehrte Mitglieder der DGKFO,

sehr geehrte Leserinnen und Leser,

nichts ist so beständig wie der Wandel. Dieses Zitat von Heraklit von Ephesos sei diesem Editorial vorangestellt, da es das letzte aus meiner Feder sein wird. Im vergangenen Jahr wurde Herr Prof. Dr. Dr. Peter Proff Präsident-elect der Deutschen Gesellschaft für Kieferorthopädie (DGKFO). Er wird das Amt des Präsidenten während der Jahrestagung 2021 übernehmen, und folgerichtig wird er Sie an dieser Stelle begrüßen.

Es hat sich seit Ende des Zweiten Weltkriegs in Deutschland eine Menge gewandelt. Dies führte vor einiger Zeit auch zur längst überfälligen Aufarbeitung der Verstrickungen von Zahnmedizinern in die NS-Ideologie im Rahmen einer ausführlichen Quellenauswertung durch den Medizinhistoriker und -ethiker Dominik Groß aus Aachen in Zahnärztliche Mitteilungen.

So umfangreich diese Texte auch waren, so gering waren doch die Berührungspunkte mit der Kieferorthopädie. Dies mag der Tatsache geschuldet sein, dass die Gebietsbezeichnungen „Fachzahnarzt für Kieferorthopädie“ und „Fachzahnarzt für Mundchirurgie“ überhaupt erst durch die Nationalsozialisten eingeführt wurden: im Jahr 1935 durch den Reichzahnärzteführer. Entsprechend wenig Kieferorthopäden dürfte es in den Folgejahren gegeben haben, sodass sie historisch nicht sinnvoll als eigene Gruppe abgegrenzt werden konnten.

Nichtsdestoweniger führten die Arbeiten von Herrn Professor Groß im Jahr 2020 zur posthumen Aberkennung der DGKFO-Ehrenmitgliedschaft von Herrn Prof. Dr. Reinhold Ritter durch die Mitgliederversammlung. In der Folgezeit wurde seitens der Mitgliedschaft der DGKFO die Rolle von Herrn Prof. Dr. Dr. Gustav Korkhaus aus Bonn, der zwei-

Prof. Dr. Jörg Lisson

joerg.lisson@uks.eu

1 Klinik für Kieferorthopädie, Universität des Saarlandes, 66421 Homburg/Saar, Deutschland felsohne auch nach der NS-Zeit eine besondere Rolle für das Fach innegehabt hatte, detaillierter hinterfragt.

Es freut mich, den Mitgliedern der DGKFO zu dieser Fragestellung einen speziellen Beitrag in der vorliegenden Ausgabe des JOO ankündigen zu dürfen, den Herr Kollege Groß exklusiv für die DGKFO verfasst hat - ausnahmsweise auf Deutsch, was an der besonderen Thematik liegt.

Mit dieser Ankündigung verabschiede ich mich nach 4 Jahren Präsidentschaft von dieser Position. Es war eine positiv bewegte Zeit für die DGKFO, in der sich sowohl für die Gesellschaft - Stichwort: Assoziation zur DGZMK als auch für das Fach - Stichwort: Kieferorthopädie als Hauptbestandteil der DMS VI - Meilensteine ergeben haben. Ganz Neugierige haben die einmalige Chance, den Studienleiter der DMS VI im Rahmen der Jahrestagung 2021 als Hauptredner zu eben diesem Thema vortragen zu hören, also noch deutlich vor der offiziellen Publikation der Ergebnisse.

Mit dem Pandemiegeschehen kam es aber auch zu erheblichen negativen Beeinträchtigungen der Gesellschaftsaktivitäten, allen voran sei hier die entfallene Jahrestagung 2020 genannt. In die Zukunft blicke ich diesbezüglich aber uneingeschränkt optimistisch.

Herrn Kollegen Proff wünsche ich einen guten Start in die neue Position. Von Ihnen wünsche ich mir, dass Sie ihm so viel positive Unterstützung zukommen lassen, wie ich sie erfahren durfte. Es war mir eine Ehre, der DGKFO als Präsident vorgestanden zu haben. Viel Freude sowie Erkenntnisgewinn bei der Lektüre des vorliegenden Heftes wünscht Ihnen

Ihr

Jörg Lisson

Präsident der DGKFO

Interessenkonflikt J. Lisson gibt an, dass kein Interessenkonflikt besteht.

Hinweis des Verlags Der Verlag bleibt in Hinblick auf geografische Zuordnungen und Gebietsbezeichnungen in veröffentlichten Karten und Institutsadressen neutral. 